\title{
Aportación de la Tomografía por Emisión de Positrones (PET) al manejo de los tumores malignos de ovario y útero
}

\author{
J. P. Suárez Fernández ${ }^{1}$, M. L. Domínguez Grande², A. Maldonado Suárez¹, L. García Bernardo², \\ M. A. Pozo García ${ }^{1}$, J. M. Alfonso Alfonso ${ }^{1}$
}

\section{Resumen}

La Tomografía por Emisión de Positrones (PET) tiene un valor limitado en el diagnóstico primario y estadificación del cáncer de ovario, aunque puede complementar a los métodos convencionales. Tampoco puede sustituir a la "second-look" dada su baja sensibilidad para detectar enfermedad micronodular. No obstante, en aquellos pacientes en los que se sospeche recurrencia, la resolución de la PET es muy alta y debe considerarse en los casos de marcador CA 125 elevado y técnicas de imágenes convencionales negativas. Los nuevos equipos híbridos PET-TAC mejoran el rendimiento diagnóstico y parece que esta técnica acabará imponiéndose a las cámaras PET dedicadas.

En el cáncer de cérvix, la PET es una técnica útil en la estadificación pre-terapéutica no invasiva de la enfermedad, ayudando a una adecuada planificación tanto quirúrgica como radioterápica. El seguimiento con PET permite un diagnóstico precoz y exacto de la enfermedad recurrente, con resultados superiores a las técnicas convencionales. Asimismo, aporta datos predictores en cuanto al pronóstico y a la respuesta a la terapia que pueden ser útiles para identificar aquellos pacientes que precisen de intervenciones más agresivas. La aplicación de la PET en el cáncer de cuerpo uterino, aunque prometedora, necesita de mayor estudio para su validación.

\section{Palabras clave:}

Tomografía por Emisión de Positrones (PET). ${ }^{18}$ F-Fluorodesoxiglucosa (FDG). Cáncer de ovario. Cáncer de cérvix. Cáncer de útero.

Oncología, 2005; 28 (5):218-231

\footnotetext{
${ }^{1}$ Centro PET Complutense (Madrid)

${ }^{2}$ Servicio de Medicina Nuclear. Hospital Universitario Infanta Cristina (Badajoz)

${ }^{3}$ Servicio de Medicina Nuclear. Hospital Central de Asturias (Oviedo)
} 


\section{Summary}

Positron Emission Tomography (PET) has a limited value in both detecting and staging ovarian cancer, although it can provide aditional information to conventional imaging. PET cannot replace the "second-look" surgical procedures because it frequently misses micronodular disease. Nevertheless, PET imaging shows a high accuracy for detecting recurrent or residual disease, so it should be recommended as the diagnostic technique of choice in these situations. The new PET-CT hybrid cameras will increase diagnostic accuracy and will probably replace dedicated PET scanners.

PET is a useful non-invasive method to assess the stage of cervical cancer prior to therapy, and may help to plan surgery or irradiation. Follow-up with PET studies allows to an early and accurate diagnosis of residual or recurrent disease with better results than conventional imaging. It can also provide useful data to predict prognosis and monitorize treatment response, in order to identify high-risk patients who will receive more aggressive therapies. Further investigations are needed to assess the value of PET in the cancer of the uterine body.

Key words: Positron Emission Tomography (PET). 18F-Fluorodeoxyglucose (FDG). Ovarian cancer. Cervical cancer. Uterine cancer.

\section{Introducción}

La Tomografía por Emisión de Positrones (PET) es una técnica de Medicina Nuclear que permite obtener imágenes de la distribución in vivo de diferentes moléculas. Es lo que se ha denominado la imagen molecular, complemento de la información anatómica que proporcionan la Tomografía Axial Computerizada (TAC) o la Resonancia Magnética (RM). Las imágenes diagnósticas de la PET se obtienen mediante la administración intravenosa de un radiofármaco, que es la unión de un isótopo radioactivo con una molécula determinada; pasados 45-60 minutos se realiza la adquisición de imágenes del cuerpo completo en una tomogammacámara PET. En la práctica clínica diaria, la molécula que se utiliza es la 2-Fluoro-D-Desoxiglucosa (FDG) que va unida al ${ }^{18} \mathrm{~F}$, isótopo radiactivo emisor de positrones de período de semidesintegración muy corto (110 minutos). La ${ }^{18} \mathrm{~F}-\mathrm{FDG}$ es un análogo de la glucosa y es captada tanto por las células normales como por las tumorales, pero no sigue el ciclo bioquímico normal de la glucosa, por lo que queda atrapada en su interior.

De forma fisiológica, algunos tejidos como el cerebro, el miocardio o el hígado tienen una alta demanda de glucosa, lo que se va a traducir en una alta retención de ${ }^{18} \mathrm{~F}-\mathrm{FDG}$. En el caso de las células malignas, su alta tasa metabólica implica igualmente una gran avidez por la ${ }^{18} \mathrm{~F}-\mathrm{FDG}$, cuyo paso al interior celular está además muy potenciado, lo que permite a priori diferenciar los tejidos malignos de los benignos. No obstante, la imagen obtenida tiene limitaciones en cuanto a la detección de la enfermedad de muy bajo grado de malignidad, debido precisamente a una menor avidez por la glucosa; del mismo modo, también puede mostrar zonas de elevada actividad celular de causa benigna, como la inflamación o la infección, que serán causas potenciales de falsos positivos. Además, aunque las tomogammacámaras PET poseen una aceptable resolución espacial (4-5 mm), cabe señalar que la imagen obtenida no será capaz de detectar la enfermedad microscópica. También estará dificultada la identificación de lesiones malignas en órganos que acumulen o eliminen de forma fisiológica la FDG, como es el caso del cerebro o los riñones.

Dado que estamos analizando el metabolismo de las células cancerosas, su detección será más precoz que las alteraciones morfológicas, parámetro utilizado por la TAC o la RM. Además, se podrán evaluar también de forma más precoz los efectos de los tratamientos tanto quimio como radioterápicos, en comparación con esas técnicas convencionales. Por 
el contrario, la falta de definición anatómica de la PET conlleva que la introducción de los nuevos equipos híbridos PET-TAC o PET-RM proporcionen al especialista una visión completa de la enfermedad, especialmente cuando se prevean procedimientos quirúrgicos.

La PET es una técnica de imagen que surge en la década de los 70 en Estados Unidos, implantándose en la práctica clínica diaria durante la década de los 90. La PET fue introducida en España en 1995 (Centro PET Complutense, Madrid). En la actualidad hay cerca de 30 centros PET distribuidos por toda la geografía española. Desde el año 2000, los hospitales públicos de las diferentes comunidades autónomas están incorporando esta tecnología a la batería de pruebas diagnósticas existentes. $\mathrm{La}{ }^{18} \mathrm{~F}$ FDG es el radiofármaco que ha permitido la introducción de la técnica PET a nivel hospitalario, a pesar de que existen en la actualidad cerca de 3.000 radiofármacos sintetizados; de aquí en adelante, se sobreentenderá al referirnos a la PET como a aquella realizada con ${ }^{18} \mathrm{~F}$-FDG.

\section{La PET en el cáncer de ovario}

El cáncer de ovario representa aproximadamente el $4 \%$ de los tumores malignos. Aunque es el cuarto cáncer más frecuente en ginecología oncológica (tras mama, endometrio y cérvix, respectivamente), sigue siendo proporcionalmente la primera causa de muerte; esto se debe a la gran dificultad de un diagnóstico precoz y a que el $65 \%$ de las pacientes se diagnostican ya en estadios avanzados (III y IV). Aunque suele haber una respuesta inicial apreciable al tratamiento, la mayoría de las pacientes desarrollan una enfermedad progresiva o recaída después de un intervalo libre de enfermedad de duración variable. El cáncer de ovario aumenta con la edad y es más frecuente en aquellas mujeres que tienen historia familiar de esta enfermedad, existiendo formas hereditarias. La asociación familiar de cáncer de ovario y de mama está relacionada en más del $90 \%$ de los casos a las mutaciones de los genes BRCA-1 y BRCA-2 ${ }^{1}$.

Los tumores ováricos derivados del epitelio y del estroma superficiales (tumores epiteliales) representan un $75 \%$ de todos los tumores del ovario y un 90\% de los cánceres de esta localización, siendo los adenocarcinomas serosos los cánceres de ovario más frecuentes (60-80\%). No ocurre lo mismo con los tumores que aparecen en mujeres menores de veinte años en las que los tumores malignos más frecuentes son de origen germinal. También existen tumores "borderline", de bajo potencial maligno, que son diferentes biológicamente de los carcinomas y no se trata de precursores de estos últimos ${ }^{2}$.

El crecimiento del cáncer epitelial de ovario es inicialmente de forma local, invadiendo la cápsula y el mesoovario, desprendiendo células a la cavidad abdominal e invadiendo la serosa de órganos pélvicos adyacentes. La afectación linfática más frecuente es la paraaórtica. Además puede aparecer una diseminación transdiafragmática, alcanzando los ganglios mediastínicos anteriores y el conducto torácico provocando un derrame pleural, hecho relativamente frecuente, siendo a veces la primera manifestación extraperitoneal del cáncer de ovario ${ }^{3}$. La diseminación hematógena del cáncer de ovario es tardía y excepcional. Las localizaciones más frecuentes son el hígado, hueso y pulmón ${ }^{4}$.

El estadiaje del cáncer de ovario se basa en la exploración clínica y quirúrgica. El estadio inicial según la clasificación FIGO/UICC ${ }^{5,6}$ y el tamaño de las lesiones residuales tras la primera cirugía, constituyen los factores pronósticos más importantes ${ }^{7}$. No obstante, entre un 30 y un $40 \%$ de carcinomas de ovario, tipificados inicialmente como estadios I o II, demuestran tener enfermedad más avanzada ${ }^{8}$. La cirugía en estadios iniciales debe incluir preceptivamente histerectomía, anexectomía bilateral, omentectomía y linfadenectomía pélvica y paraaórtica9. En estadios avanzados, se realiza cirugía citorreductora, seguida de tratamiento quimioterápico ${ }^{10}$. El cáncer de ovario es generalmente quimiosensible, aunque desarrolla rápidamente resistencias a los tratamientos. Los agentes quimioterápicos más activos en el cáncer de ovario son los derivados del platino y los taxanos. Cuando éstos fracasan o se producen recidivas, se utilizan fármacos de segunda línea. A pesar de que la respuesta a tratamientos de segunda línea no es inusual, las respuestas tienden a ser breves y las supervivencias de larga duración son ra$\operatorname{ras}^{11}$. El seguimiento de las pacientes se realiza fundamentalmente mediante exploración clínica, TAC y ecografía abdómino-pélvica y determinación sérica de CA-125.

El mecanismo de captación de FDG por las célu- 
las tumorales ováricas depende, al igual que en otras neoplasias, de la expresión de los transportadores de membrana de glucosa (especialmente el GLUT-1), del índice proliferativo tumoral (MIB-1) y del grado histológico, sin que se haya demostrado una correlación con el estadio clínico ${ }^{12}$. Se han descrito varias situaciones en las que la PET con FDG puede aportar información útil para el manejo de las pacientes con cáncer de ovario, que detallaremos a continuación.

\section{Diagnóstico primario de malignidad}

Como se ha expuesto anteriormente, el cáncer de ovario suele detectarse en estadios avanzados debido a la ausencia de síntomas precoces. Cuando se detecta una masa anexial asintomática, surge la necesidad de caracterizar su origen benigno o maligno. El antígeno CA-125 se encuentra elevado en el $80 \%$ de los cánceres de ovario, pero su sensibilidad es mucho menor en estadios iniciales y puede elevarse en algunas enfermedades benignas, en tumores benignos ginecológicos, incluídos los del ovario, y en neoplasias malignas no ováricas ${ }^{13}$. La ecografía es la técnica más sensible en la detección de masas anexiales, pero su especificidad es limitada ${ }^{14}$. La TAC y la RM informan de la resecabilidad y extensión regional y a distancia del tumor, y pueden diagnosticar tumores benignos quísticos (quistes dermoides y en- dometriales). No obstante, les sigue faltando una adecuada especificidad en el resto de los casos ${ }^{15,16}$.

La PET obtiene unas cifras de sensibilidad comparables a las de la TAC (50-90\%), pero con mayor especificidad (60-80\%) (Tabla I). No obstante, es incapaz de detectar la mayor parte de los tumores en estadio I y aquellos de malignidad "borderline", al correlacionarse la captación de FDG con el grado histológico ${ }^{19,21}$. Además, se pueden producir falsos positivos en lesiones benignas tales como endometriosis, tumores quísticos, teratomas, schwanomas, enfermedades inflamatorio-infecciosas y hemorragias del cuerpo lúteo. El análisis semicuantitativo del SUV (Standard Value Uptake) no parece que aumente la especificidad ${ }^{22,24}$. La presencia de captación fisiológica en el intestino y en las vías urinarias y en la vejiga puede confundir en muchas ocasiones la interpretación de los estudios. Algunos autores recomiendan sondaje urinario con irrigación continua para minimizar este problema ${ }^{25}$. El estado hormonal de la paciente también es un dato a tener en cuenta, ya que es posible encontrar captación fisiológica ovárica en pacientes premenopáusicas ${ }^{26}$. Está por determinar si la realización de imágenes tardías podría aumentar la exactitud de la técnica, como se ha comprobado en otros tumores. Otros trazadores, como la ${ }^{11} \mathrm{C}$-Metionina, no obtienen mejores resultados que la FDG, aunque podrían detectar más fácilmente tumores de malignidad "borderline" al no depender

TABLA I

Resultados de la PET con FDG en el diagnóstico diferencial de masas ováricas

\begin{tabular}{|c|c|c|c|c|c|c|c|c|c|c|c|}
\hline$R$ & Autor (año) & $N$ & $\begin{array}{l}\text { SEN } \\
\text { PET }\end{array}$ & $\begin{array}{l}E S P \\
P E T\end{array}$ & $\begin{array}{l}E D \\
P E T\end{array}$ & $\begin{array}{l}V P P \\
P E T\end{array}$ & $\begin{array}{l}V P N \\
P E T\end{array}$ & $\begin{array}{c}S E N \\
M D C\end{array}$ & $\begin{array}{c}E S P \\
M D C\end{array}$ & $\begin{array}{c}E D \\
M D C\end{array}$ & $\begin{array}{c}\text { Gold } \\
\text { Standard }\end{array}$ \\
\hline 17 & Hubner (1993) & 51 & - & - & - & $86 \%$ & $76 \%$ & - & - & - & $\mathrm{CH}$ \\
\hline 16 & Römer (1997) & 19 & $83 \%$ & $54 \%$ & - & $46 \%$ & $88 \%$ & - & - & - & $\mathrm{CH}$ \\
\hline 24 & Zimny (1997) & 26 & $88 \%$ & $80 \%$ & $85 \%$ & - & - & - & - & - & $\mathrm{CH}$ \\
\hline 19 & Schröder (1999) & 24 & $79 \%$ & $90 \%$ & $86 \%$ & $92 \%$ & $75 \%$ & - & - & - & $\mathrm{CH}$ \\
\hline 16 & Rieber (2001) & 103 & $58 \%$ & $78 \%$ & $76 \%$ & - & - & $\begin{array}{l}83 \%^{1} \\
92 \%^{2}\end{array}$ & $\begin{array}{l}84 \%^{1} \\
59 \%^{2}\end{array}$ & $\begin{array}{l}83 \%^{1} \\
63 \%^{2}\end{array}$ & $\mathrm{CH}$ \\
\hline 20 & Liebermann*(2001) & 17 & $90 \%$ & $71 \%$ & - & $82 \%$ & $83 \%$ & - & - & - & $\mathrm{CH}$ \\
\hline 21 & Fenchel (2002) & 99 & $58 \%$ & $76 \%$ & - & $25 \%$ & $93 \%$ & - & - & - & $\mathrm{CH}$ \\
\hline
\end{tabular}

R, Referencia; N, no pacientes; SEN, Sensibilidad; ESP, Especificidad; ED, Exactitud diagnóstica; VPP, Valor predictivo positivo; VPN, Valor predictivo negativo; MDC, Métodos de diagnóstico convencionales; CH, Confirmación histológica; RM, Resonancia magnética.

\footnotetext{
* Estudio realizado con gammacámara de coincidencia.

${ }^{1} \mathrm{RM}$

${ }^{2}$ Ecografía transvaginal
} 
tanto del grado histológico ${ }^{23}$. También se ha ensayado con ${ }^{11} \mathrm{C}$-Colina, que presenta la ventaja de que su eliminación urinaria es prácticamente nula ${ }^{27}$.

La RM tiene una buena eficacia diagnóstica en cáncer de ovario, y se ha sugerido que la combinación de imágenes PET y RM obtendrían sensibilidades cercanas a la ecografía con mayor especifici$\mathrm{dad}^{16}$. Cabe augurar que la reciente introducción de equipos híbridos PET-TAC mejore la detección de lesiones malignas al combinar imagen anatómica y metabólica. Mientras tanto, la ecografía y la exploración quirúrgica seguirán siendo los métodos de elección para la detección y caracterización del cáncer ovárico.

\section{Estadificación tumoral pre-terapéutica}

La estadificación definitiva de un cáncer de ovario viene determinada tras la cirugía de resección tumoral con exploración de la cavidad peritoneal, lavado y toma de biopsias múltiples, como ya hemos comentado. Con la intención de que esta cirugía sea curativa, es importante conocer de antemano la posible extensión extrapélvica de la enfermedad mediante métodos no invasivos. La PET obtiene cifras aceptables de sensibilidad en la estadificación ganglionar con muy alta especificidad. Los falsos negativos provienen fundamentalmente de la falta de resolución espacial, no detectándose adenopatías metastásicas menores de 4-5 mm ${ }^{19}$, 20. 24 . Aquí nuevamente podría jugar un papel importante la imagen híbrida PET-TAC, ya que en algunos estudios se ha constatado una mejora en la exactitud de la estadificación preterapéutica combinando ambas técnicas ${ }^{28}$. Es sabido, además, que la TAC y la RM no pueden descartar implantes peritoneales de pequeño tamaño. La PET obtiene en estos casos una sensibilidad y especificidad del $50-78 \%$ y $86-95 \%{ }^{19}, 24$, respectivamente, cifras superiores a las obtenidas por las técnicas convencionales. Frecuentemente se escapan a la detección lesiones microscópicas o micronodulares $^{24,29}$. En estos casos de siembra peritoneal de pequeño tamaño, puede observarse en la imagen PET un patrón inespecífico de aumento difuso de la captación abdominal de $\mathrm{FDG}^{30}$.

Otra ventaja de la PET es que puede descartar metástasis ganglionares alejadas o hematógenas en tumores muy avanzados, al poder visualizar todo el cuerpo en una única exploración (Fig. 1).

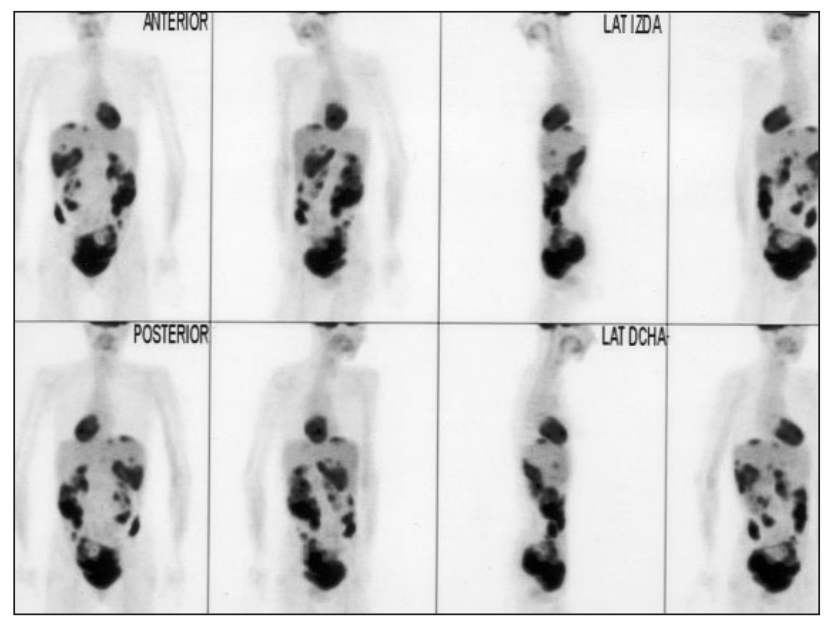

Figura 1. Cáncer de ovario izquierdo localmente avanzado (cistadenocarcinoma papilar seroso), mostrando una captación irregular típica con zonas quísticas hipometabólicas en su interior. Se puede apreciar una extensa carcinomatosis peritoneal que afecta a prácticamente todo el abdomen, incluído el espacio subhepático.

\section{Diagnóstico de enfermedad residual o recurrente}

Son las 2 indicaciones clínicas en las que la PET obtiene un mayor rendimiento diagnóstico. Una de las situaciones estudiadas es si la PET podría sustituir a la "second-look" en pacientes con remisión completa clínica y radiológica y con niveles normales de CA-125. En este sentido, varios estudios (Tabla II) han constatado la incapacidad de la técnica para detectar enfermedad de menos de 4-5 mm., con unas cifras globales de sensibilidad y especificidad de $50-70 \%$ y $70-90 \%$, respectivamente (valor predictivo negativo de aproximadamente $50 \%$ ). Por ello, la laparotomía exploradora no podrá ser sustituida por la PET, en los casos en que se considere necesaria pese a que aún existe controversia sobre si mejora o no la supervivencia. No obstante, un estudio PET positivo puede aportar información pronóstica, ya que sus hallazgos preceden en varios meses la aparición de síntomas o imágenes sospechosas por otros métodos de imagen (Fig. 2). Por otro lado, el intervalo libre de enfermedad (ILE) de las pacientes con una PET negativa es casi 2 años mayor que las que tienen una PET positiva ${ }^{34}$. Esto tiene implicaciones terapéuticas, ya que las pacientes con un ILE mayor de 6 meses serían potencialmente respondedoras a los tratamientos basados en derivados del platino, y sobre todo se beneficiarían del re-tra- 
TABLA II

Resultados de la PET con FDG en el diagnóstico de enfermedad residual o recurrente en el cáncer de ovario

\begin{tabular}{|c|c|c|c|c|c|c|c|c|c|c|}
\hline$R$ & Autor (año) & $N$ & Indicación & $\begin{array}{l}\text { SEN } \\
P E T\end{array}$ & $\begin{array}{l}E S P \\
P E T\end{array}$ & $\begin{array}{l}E D \\
P E T\end{array}$ & $\begin{array}{l}S E N \\
M D C\end{array}$ & $\begin{array}{c}E S P \\
M D C\end{array}$ & $\begin{array}{c}E D \\
M D C\end{array}$ & $\begin{array}{c}\text { Gold } \\
\text { Standard }\end{array}$ \\
\hline 31 & Jiménez (2000) & 14 & SR & $100 \%$ & $50 \%$ & $93 \%$ & - & - & - & $\mathrm{CH}$ y EC \\
\hline 32 & Rose (2001) & 22 & LE & $10 \%$ & $42 \%$ & - & - & - & - & $\mathrm{CH}$ \\
\hline 33 & Nakamoto (2001) & 24 & $\begin{array}{l}\text { SR (12) } \\
\text { LE (12) }\end{array}$ & $\begin{array}{l}80 \% \\
66 \%\end{array}$ & $\begin{array}{l}50 \% \\
89 \%\end{array}$ & & $73 \%$ & $75 \%$ & $73 \%$ & $\mathrm{CH}$ y EC \\
\hline 34 & Zimny (2001) & 54 & $\begin{array}{l}\text { SR } \\
\text { LE }\end{array}$ & $\begin{array}{l}94 \% \\
65 \%\end{array}$ & $\begin{array}{l}75 \% \\
83 \%\end{array}$ & $83 \%$ & - & - & - & $\mathrm{CH}$ y EC \\
\hline 35 & Torizuka (2002) & 25 & SR & $80 \%$ & $100 \%$ & $84 \%$ & $\begin{array}{l}55 \%^{3} \\
75 \%^{4}\end{array}$ & $\begin{array}{l}100 \% \%^{3} \\
100 \%{ }^{4}\end{array}$ & $\begin{array}{l}64 \%^{3} \\
80 \%^{4}\end{array}$ & $\mathrm{CH}$ y EC \\
\hline 36 & Chang (2002) & 28 & SR & $95 \%$ & $88 \%$ & $93 \%$ & - & - & - & $\mathrm{CH}$ y EC \\
\hline 37 & Cho (2002) & 31 & LE & $45 \%$ & $99 \%$ & $91 \%$ & $54 \%^{3}$ & $99 \%^{3}$ & $92 \%^{3}$ & $\mathrm{CH}$ \\
\hline 38 & García (2003) & 19 & SR & $100 \%$ & - & $90 \%$ & $\begin{array}{l}47 \%^{3} \\
84 \%^{4}\end{array}$ & $\begin{array}{l}- \\
-\end{array}$ & $\begin{array}{l}43 \%^{3} \\
86 \%^{3}\end{array}$ & $\mathrm{CH}$ y EC \\
\hline 39 & Picchio ${ }^{1}(2003)$ & 25 & $\begin{array}{c}\text { Previo a } \\
\text { second-look }\end{array}$ & $83 \%$ & $92 \%$ & $95 \%$ & $\begin{array}{l}70 \%^{3} \\
62 \%^{4}\end{array}$ & $\begin{array}{l}83 \%^{3} \\
83 \%^{4}\end{array}$ & $\begin{array}{l}74 \%^{3} \\
72 \% \%^{4}\end{array}$ & $\mathrm{CH}$ \\
\hline 40 & Bristow $^{2}$ (2003) & 22 & SR & $83 \%$ & $75 \%$ & $82 \%$ & - & - & - & $\mathrm{CH}$ \\
\hline 41 & Sironi $^{2}(2004)$ & 31 & $\begin{array}{c}\text { Previo a } \\
\text { second-look }\end{array}$ & $78 \%$ & $75 \%$ & $77 \%$ & - & - & - & $\mathrm{CH}$ \\
\hline
\end{tabular}

R, Referencia; N, nº pacientes; SEN, Sensibilidad; ESP, Especificidad; ED, Exactitud diagnóstica; SR, Sospecha de recurrencia; LE, Pacientes libres de enfermedad; MDC, Métodos de diagnóstico convencionales; $\mathrm{CH}$, Confirmación histológica; EC, Evolución clínica; TAC, Tomografía axial computerizada; RM, Resonancia magnética.

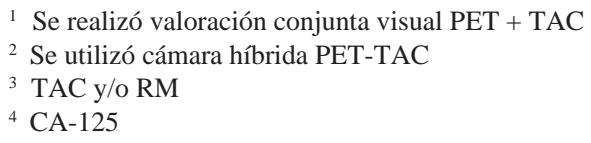

tamiento si el ILE es superior a 24 meses. Se ha sugerido que la PET podría ser una alternativa a la "second-look" en pacientes con tumores avanzados que presenten alteraciones en TAC o elevación de marcadores tumorales, actitud que sería muy positiva en términos de coste-efectividad ${ }^{42}$.

Otra situación frecuente se da en aquellas pacien-

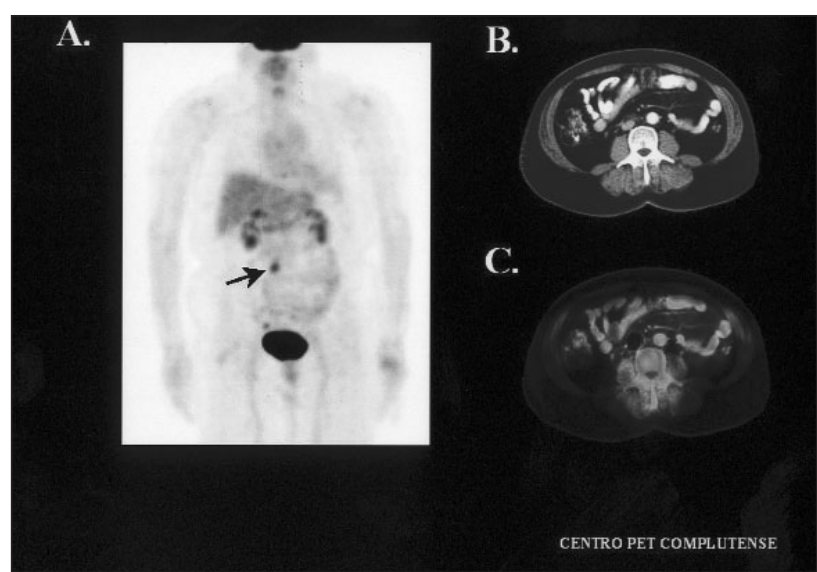

tes que alcanzaron una respuesta completa tumoral y en las que durante el seguimiento se objetiva la reaparición de sintomatología, un aumento de marcadores tumorales (CA-125), y/o alteraciones en las pruebas de imagen. En estos casos, y sobre todo cuando la elevación aislada del CA-125 es el único dato que apoya la sospecha de recidiva, la PET obtiene resultados mucho mejores, con sensibilidad y especificidad rondando el 90-95\% (Tabla II). Algunos estudios se han realizado con las nuevas cámaras híbridas PET-TAC, demostrándose mayor exactitud que con ambas técnicas por separado ${ }^{40,41}$.

La caracterización de masas residuales tras el tratamiento es otra de las posibles indicaciones dentro de este ámbito, cuya utilidad está sobradamente demostrada en tumores de otras áreas.

Figura 2. Paciente con cáncer de ovario tratado mediante cirugía y quimioterapia en remisión completa hasta que se detectan 2 adenopatías retroperitoneales en TAC de control. La PET confirma enfermedad tumoral activa en una de ellas (A., flecha). Detalle del corte TAC transaxial a dicho nivel (B.) y de la fusión PET-TAC (C.). 


\section{Monitorización de la respuesta al tratamiento}

Habitualmente, los criterios de respuesta a un tratamiento son anatómicos, presuponiendo que una disminución del tamaño tumoral indica que dicha terapia es eficaz. No obstante, aunque no se objetiven cambios morfológicos, puede haber una respuesta de grado variable que no sea evidente hasta pasadas varias semanas. La PET-FDG tiene la ventaja sobre las técnicas de imagen convencionales de que permite evaluar el descenso metabólico los focos tumorales que responden a un determinado tratamiento, por lo cual obtenemos información más precoz y exacta sobre la eficacia del mismo, ya que precede a los cambios de tamaño. Esto se ha observado en varios tipos de tumores, pero no existen estudios amplios en el caso concreto del cáncer de ovario. No obstante, Zimny y cols. ${ }^{24}$ mostraron en 6 pacientes cómo la captación de FDG en implantes peritoneales y metástasis linfáticas disminuía paralelamente a los niveles de CA-125 a las 3 semanas del inicio del tratamiento quimioterápico.

\section{La PET en el cáncer de útero}

\subsection{Cáncer de cérvix}

La mortalidad por cáncer de cérvix ha disminuido en los últimos años de una forma global gracias a la introducción de métodos de cribaje efectivos de la enfermedad pre-invasiva, como la citología cérvicovaginal. No obstante, esta reducción no ha afectado a los pacientes con tumores avanzados al diagnóstico. El $80-90 \%$ de los tumores malignos de cérvix son escamosos, por lo que su comportamiento es inicialmente la invasión local (útero, vagina, parametrios, ligamentos uterinos) y linfática (ganglios pélvicos y posteriormente cadena paraaórtica). Las metástasis hematógenas son poco comunes y rara vez aparecen en estadios tempranos. En cambio, los tumores cervicales de estirpe no escamosa (adenocarcinomas) tienen un mayor riesgo de extenderse por vía sanguínea. Los factores pronósticos más importantes son el tamaño tumoral y la presencia o no de afectación ganglionar. El tratamiento en los estadios iniciales puede ser quirúrgico y/o radioterápico, con similares resultados. En estadios avanzados, la combinación de radio-quimioterapia suele ser la mejor opción para el control tumoral.
Como ocurre con tumores escamosos y adenocarcinomas de otras localizaciones, el cáncer de cérvix acumula con avidez la FDG, lo que permite su evaluación mediante PET. Lógicamente, al ser el diagnóstico inicial fundamentalmente clínico y citológico, no se ha evaluado la PET como método de caracterización de masas del cuello uterino. En cambio, sí poseemos datos sobre su utilidad en la estadificación pre-terapéutica, en la detección de enfermedad residual o recurrente, en la valoración de la respuesta a los tratamientos y en cuanto su valor pronóstico.

\section{Estadificación tumoral pre-terapéutica}

Los métodos de imagen anatómicos se han utilizado sistemáticamente para establecer el grado de invasión local y regional de los carcinomas de cérvix. La RM es la prueba que se ha mostrado más efectiva para detectar la infiltración útero-vaginal y parametrial, al visualizar el estado de los tejidos blandos con mayor nitidez que la TAC. En este sentido, la PET es poco útil al carecer de una adecuada resolución espacial, aspecto que posiblemente vendrá mejorado con las cámaras híbridas PET-TAC. La estadificación ganglionar también se basa en los criterios morfológicos aportados por TAC y RM. Su sensibilidad y especificidad varían entre $38-89 \%$ y $78-99 \%$, respectivamente $^{43}$. Esta falta de sensibilidad implica que muchos cánceres de cérvix sean infraestadificados.

La PET obtiene cifras de sensibilidad entre el 75$100 \%$ y de especificidad entre $92-100 \%$, superiores en la mayoría de los casos a las técnicas de imagen convencionales (Tabla III). La PET detecta mejor la afectación ganglionar pélvica que la paraórtica ${ }^{45,47}$, algo que puede ser paradójico dadas las limitaciones debidas a la actividad fisiológica urinaria e intestinal; como la PET no puede detectar enfermedad menor de 5-10 mm, es posible que este hecho se deba a que pequeños ganglios recientemente afectados y con micrometástasis escapen a la detección, ya que como hemos comentado, la afectación retroperitoneal es temporalmente posterior a la invasión ganglionar pélvica ${ }^{51}$. En la detección de afectación ganglionar a distancia, como en los huecos supraclaviculares, es en cambio una técnica muy efectiva, localizando lesiones no sospechadas en muchos casos y cambiando la actitud terapéutica en un porcentaje significativo $^{52,53}$ (Fig. 3). En ese último aspecto, cabe destacar la utilidad de la PET para mejorar la pla- 
TABLA III

Resultados de la PET con FDG en la estadificación pre-terapéutica del cáncer de cérvix

\begin{tabular}{|c|c|c|c|c|c|c|c|c|c|c|c|}
\hline$R$ & Autor (año) & $N$ & $\begin{array}{l}\text { SEN } \\
P E T\end{array}$ & $\begin{array}{l}E S P \\
P E T\end{array}$ & $\begin{array}{l}E D \\
P E T\end{array}$ & $\begin{array}{l}V P P \\
P E T\end{array}$ & $\begin{array}{l}V P N \\
P E T\end{array}$ & $\begin{array}{l}S E N \\
M D C\end{array}$ & $\begin{array}{l}E S P \\
M D C\end{array}$ & $\begin{array}{c}E D \\
M D C\end{array}$ & $\begin{array}{c}\text { Gold } \\
\text { Standard }\end{array}$ \\
\hline 44 & Sugawara (1999) & 17 & $86 \%$ & - & - & - & - & $57 \%^{1}$ & - & - & $\mathrm{CH}$ parcial \\
\hline 45 & Rose (1999) & 32 & $\begin{array}{l}100 \%^{4} \\
75 \% \%^{3}\end{array}$ & $\begin{array}{l}100 \% \%^{4} \\
92 \%^{3}\end{array}$ & $\begin{array}{l}100 \%^{4} \\
83 \%^{3}\end{array}$ & $\begin{array}{l}100 \%^{4} \\
75 \%^{3}\end{array}$ & $\begin{array}{l}100 \% \%^{4} \\
92 \%{ }^{3}\end{array}$ & $50 \%^{1}$ & - & - & $\mathrm{CH}$ \\
\hline 46 & Reinhardt (2001) & 35 & $91 \%$ & $100 \%$ & - & $100 \%$ & - & $73 \%{ }^{2}$ & $83 \%^{2}$ & - & $\mathrm{CH}$ \\
\hline 47 & Norayan (2001) & 27 & $\begin{array}{l}83 \%^{4} \\
57 \%^{3}\end{array}$ & $92 \%{ }^{4}$ & $88 \%{ }^{4}$ & $91 \%{ }^{4}$ & $85 \%{ }^{4}$ & $50 \%{ }^{2}$ & - & $75 \%{ }^{2}$ & $\mathrm{CH}$ \\
\hline 48 & Yeh (2002) & 42 & $83 \%$ & $97 \%$ & $93 \%$ & - & - & \multicolumn{3}{|c|}{$\begin{array}{l}\text { RM negativa o } \\
\text { indeterminada }\end{array}$} & $\mathrm{CH}$ \\
\hline 49 & Ma (2003) & $39^{3}$ & $\begin{array}{c}82 \% \\
100 \% \text { TI }\end{array}$ & $\begin{array}{c}97 \% \\
100 \%\end{array}$ & $\begin{array}{c}91 \% \\
100 \%\end{array}$ & $\begin{array}{c}94 \% \\
100 \%\end{array}$ & $\begin{array}{c}90 \% \\
100 \%\end{array}$ & $76 \% \%^{1-2}$ & $97 \% \%^{1-2}$ & $89 \% \%^{1-2}$ & $\mathrm{CH}$ y EC \\
\hline 50 & Lin (2003) & 50 & $86 \%$ & $94 \%$ & $92 \%$ & - & - & \multicolumn{3}{|c|}{$\begin{array}{l}\text { TAC negativo o } \\
\text { indeterminado }\end{array}$} & $\mathrm{CH}$ \\
\hline
\end{tabular}

R, Referencia; N, $n^{\circ}$ pacientes; SEN, Sensibilidad; ESP, Especificidad; ED, Exactitud diagnóstica; VPP, Valor predictivo positivo; VPN, Valor predictivo negativo; MDC, Métodos de diagnóstico convencionales; CH, Confirmación histológica; EC, Evolución clínica; TAC, Tomografía axial computerizada; RM, Resonancia magnética.

${ }^{1} \mathrm{TAC} ;{ }^{2} \mathrm{RM} ;{ }^{3}$ Estadificación ganglionar paraaórtica; ${ }^{4}$ Estadificación ganglionar pélvica

II Imagen PET-FDG tardía (3 horas post-inyección)

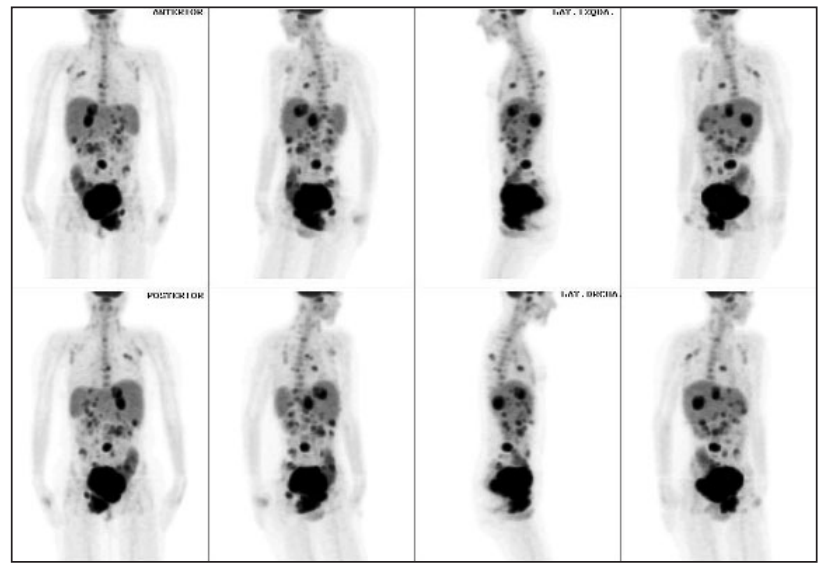

Figura 3. Paciente que acude tras conización por carcinoma de cérvix. El estudio de estadificación con PET-FDG muestra afectación neoplásica múltiple en hueso, mediastino, hígado, retroperitoneo, peritoneo abdómino-pelviano y útero.

nificación radioterápica, respaldado por algunos trabajos $^{54-56}$. Algunos autores defienden que la realización de imágenes tardías a las 3 horas de la inyección de la FDG mejoraría globalmente la exactitud de la técnica ${ }^{49,57,58}$. Sea como fuere, es previsible que la imagen híbrida PET-TAC suponga un mayor avance en la mejora de la estadificación no invasiva.

\section{Diagnóstico de enfermedad residual o recurrente}

Las técnicas de imagen anatómicas tienen dificultades en la detección de tejido tumoral residual debido su incapacidad para distinguirlo con exactitud de los cambios producidos por los tratamientos aplicados, sobre todo tras la irradiación. Del mismo modo, pueden dar resultados negativos o equívocos cuando existe una recidiva tumoral, lo que crea incertidumbre a la hora de tomar decisiones, sobre todo si existen datos clínicos o bioquímicos (elevación del antígeno de células escamosas -SCC-) que apoyen esta sospecha.

En estas situaciones, la PET presenta sensibilidades entre $80-100 \%$ y especificidades entre $76-100 \%$ (Tabla IV). Permite la caracterización metabólica de masas residuales post-tratamiento con alta exactitud y de forma precoz, y puede servir como guía para la confirmación histológica mediante biopsia o punción, por lo que puede tener un impacto considerable en el manejo terapéutico ${ }^{63,67-69}$. Pueden producirse resultados falsos positivos debido a fenómenos inflamatorios, sobre todo tras la radioterapia, por lo que se recomienda que al menos hayan pasado un mínimo de 2 meses tras la irradiación antes de realizar el 
TABLA IV

Resultados de la PET con FDG en la detección de enfermedad residual y recurrente en cáncer de cérvix

\begin{tabular}{|c|c|c|c|c|c|c|c|c|c|c|c|}
\hline$R$ & Autor (año) & $N$ & $\begin{array}{l}\text { SEN } \\
\text { PET }\end{array}$ & $\begin{array}{l}\text { ESP } \\
P E T\end{array}$ & $\begin{array}{l}E D \\
P E T\end{array}$ & $\begin{array}{l}V P P \\
P E T\end{array}$ & $\begin{array}{l}V P N \\
P E T\end{array}$ & $\begin{array}{c}S E N \\
M D C\end{array}$ & $\begin{array}{l}E S P \\
M D C\end{array}$ & $\begin{array}{c}E D \\
M D C\end{array}$ & $\begin{array}{c}\text { Gold } \\
\text { Standard }\end{array}$ \\
\hline 59 & Park (2000) & 36 & $100 \%$ & $94 \%$ & $97 \%$ & - & - & $78 \%$ & $83 \%$ & $81 \%$ & $\mathrm{CH}$ y EC \\
\hline 60 & Sun (2001) & 20 & $90 \%$ & $100 \%$ & - & - & - & - & - & - & $\mathrm{CH}$ y $\mathrm{EC}$ \\
\hline 61 & Ryu (2003) & 249 & $93 \%$ & $76 \%$ & - & - & - & - & - & - & $\mathrm{CH}$ y $\mathrm{EC}$ \\
\hline 62 & Havrilesky (2003) & 28 & $86 \%$ & $87 \%$ & - & $86 \%$ & $87 \%$ & - & - & - & $\mathrm{CH}$ y EC \\
\hline 49 & Ma (2003) & $65^{3}$ & $\begin{array}{c}82 \% \\
100 \% \text { II }\end{array}$ & $\begin{array}{c}97 \% \\
100 \% \pi\end{array}$ & $\begin{array}{l}91 \% \\
100 \%{ }^{\mathbb{T}}\end{array}$ & $\begin{array}{c}94 \% \\
100 \%{ }^{\mathbb{I}}\end{array}$ & $\begin{array}{c}90 \% \\
100 \% \pi\end{array}$ & $76 \% \%^{1-2}$ & $97 \%^{1-2}$ & $89 \% \%^{1-2}$ & $\mathrm{CH}$ y EC \\
\hline 63 & Lai (2004) & 40 & $92 \%$ & - & - & - & - & $60 \% \%^{1-2}$ & - & - & $\mathrm{CH}$ y EC \\
\hline 64 & Wong (2004) & 35 & $82-100 \%$ & $90-97 \%$ & $92-94 \%$ & - & - & - & - & - & $\mathrm{CH}$ y $\mathrm{EC}$ \\
\hline 65 & Chang (2004) & $27^{4}$ & $94 \%$ & $78 \%$ & $89 \%$ & $89 \%$ & $87 \%$ & - & - & - & $\mathrm{CH}$ y EC \\
\hline 66 & Unger (2004) & 44 & $80-100 \%$ & $86-100 \%$ & - & $93-100 \%$ & $89-100 \%$ & - & - & - & $\mathrm{CH}$ y $\mathrm{EC}$ \\
\hline
\end{tabular}

R, Referencia; N, nº pacientes; SEN, Sensibilidad; ESP, Especificidad; ED, Exactitud diagnóstica; VPP, Valor predictivo positivo; VPN, Valor predictivo negativo; MDC, Métodos de diagnóstico convencionales; CH, Confirmación histológica; EC, Evolución clínica; TAC, Tomografía axial computerizada; RM, Resonancia magnética; SCC, Antígeno de células escamosas.

${ }^{1} \mathrm{TAC} ;{ }^{2} \mathrm{RM} ;{ }^{3}$ Estadificación ganglionar paraaórtica; ${ }^{4}$ Elevación SCC

II Imagen PET-FDG tardía (3 horas post-inyección)

estudio PET. Los falsos negativos, menos frecuentes, son debidos a la presencia de volúmenes tumorales de tamaño inferior a 5-10 mm, aunque la actividad urinaria fisiológica también puede limitar la capacidad de detección de lesiones de mayor tamaño.

Las recidivas tumorales suelen ser locorregionales, más frecuentes tras la radioterapia, y pueden sospecharse clínicamente, por alteración de las pruebas de imagen convencionales o por elevaciones séricas del SCC. La PET es capaz de confirmar lesiones sospechosas, y de detectar y localizar recidivas no sospechadas, tanto regionales como a distancia, lo que la convierten en una alternativa atractiva para el seguimiento de estas pacientes ${ }^{59-69}$ (Fig. 4).

\section{Monitorización de la respuesta al tratamiento}

Tal y como se ha expuesto para el cáncer de ovario, la PET puede ser útil para identificar de forma precoz aquellas pacientes en las que el tratamiento es eficaz, sobre todo en estadios avanzados en los que se combina radio y quimioterapia. Al contrario de lo que ocurre con la radioterapia, donde el efecto de la radiación sobre los tejidos neoplásicos y sanos provoca reacciones inflamatorias que no permiten la valoración con PET hasta pasados 2-3 meses, debido a la alta probabilidad de falsos positivos, la quimioterapia no

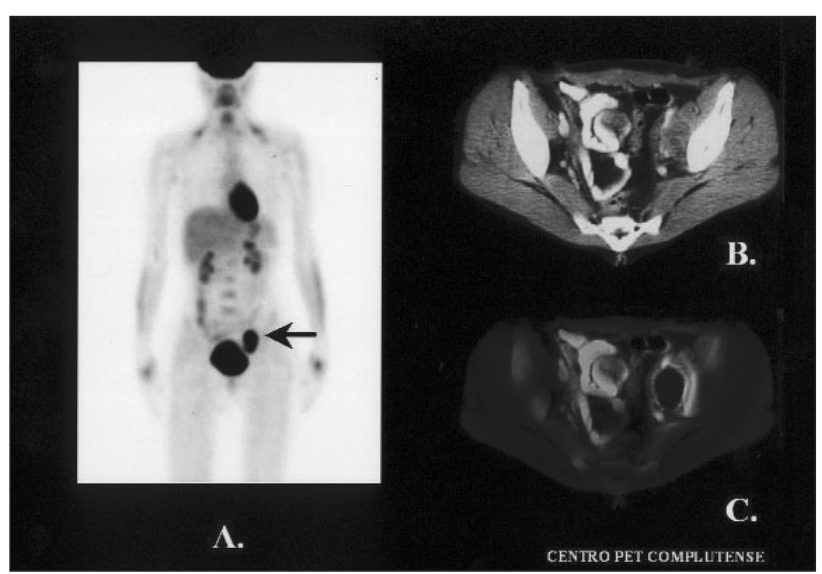

Figura 4. Paciente con carcinoma epidermoide de cérvix intervenido mediante histerectomía total más doble anexectomía. En TAC se detecta un conglomerado de adenopatías en cadena ilíaca común izquierda que se propaga caudalmente por la cadena ilíaca interna. Los marcadores tumorales son normales. La PET detecta la afectación ganglionar maligna (A., flecha). Detalle del corte TAC transaxial a dicho nivel (B.) y de la fusión PET-TAC (C.).

presenta dicho problema, pudiendo ser valorada su efectividad pocos días después del primer ciclo. Otra ventaja de la PET es que ofrece resultados sobre la efectividad de la quimioterapia de forma más rápida que el resto de técnicas de imagen, información que permite modificar los tratamientos precozmente en 
los pacientes no respondedores, si fuera preciso. Para ello se valorará no sólo la disminución visual de tamaño y de intensidad de captación con respecto al estudio basal, sino también una reducción semicuantitativa mediante la determinación del SUV. Así, según las recomendaciones del grupo PET de la European Organization for Research and Treatment of Cancer (EORTC PET Group), una disminución del SUV menor del $15 \%$ indicaría enfermedad estable, mientras que una reducción del 15-25\% tras el primer ciclo de quimioterapia o mayor del $25 \%$ tras 2 o más ciclos, indicaría una respuesta metabólica parcial; y las respuestas completas implicarían una total desaparición de la captación de FDG del volumen tumoral ${ }^{70}$.

Los pocos trabajos publicados hasta la fecha se centran en la valoración de la respuesta tumoral a la radioterapia. Nakamoto y cols. ${ }^{71}$ monitorizaron 20 pacientes mediante estudios PET pre y post-radioterapia, obteniendo una sensibilidad, especificidad y exactitud del 100\%, 60\% y 70\%, respectivamente. Un estudio retrospectivo más reciente de Grigsby y cols. $^{72}$ sobre 152 pacientes tratadas con radioterapia y cisplatino concomitante concluyó que la PET es el factor predictor más importante de recurrencia y supervivencia en el cáncer de cérvix.

\section{La PET como factor pronóstico}

El grado de acumulación de FDG por parte de los tumores malignos puede ser un parámetro de la agresividad tumoral que puede servir para predecir el control local y la remisión completa tras tratamientos. Elevadas captaciones en el cáncer de cérvix (SUV>13) parecen indicar un peor pronóstico y la necesidad de aplicar terapias más agresivas ${ }^{73}$. También se ha sugerido el volumen tumoral medido por PET como otro factor predictor de la supervivencia $^{74}$. No obstante, la ausencia de afectación ganglionar paraaórtica en el estudio PET se ha mostrado como el factor pronóstico más significativo de supervivencia, según el análisis multivariante realizado por Grigsby y cols. ${ }^{75}$.

\subsection{Cáncer de cuerpo uterino}

Ningún estudio hasta la fecha ha abordado la utilidad de la PET en el diagnóstico diferencial de masas uterinas ni en la estadificación pre-terapéutica de los tumores malignos de esta localización. Sólo el

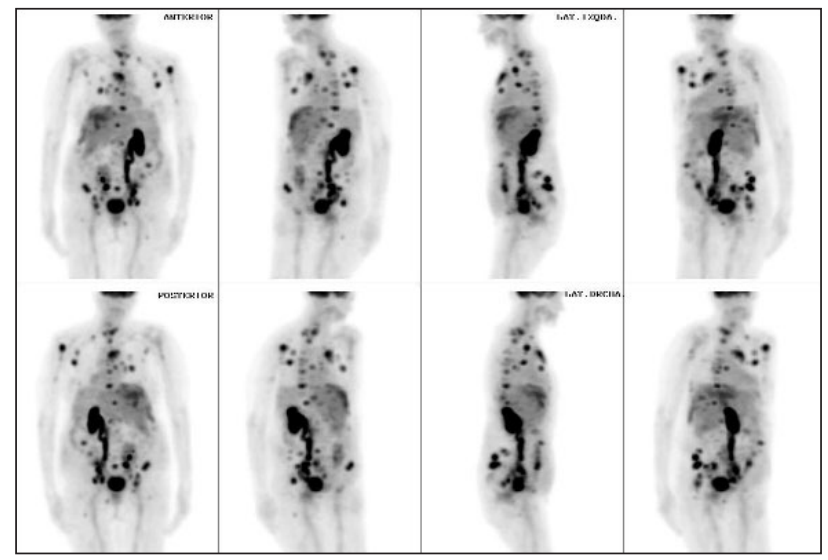

Figura 5. Adenocarcinoma de endometrio intervenido y tratado con radioterapia. Actualmente se detecta metástasis en tejido celular subcutáneo. PET-FDG: Afectación neoplásica múltiple ósea, mediastínica y peritoneal.

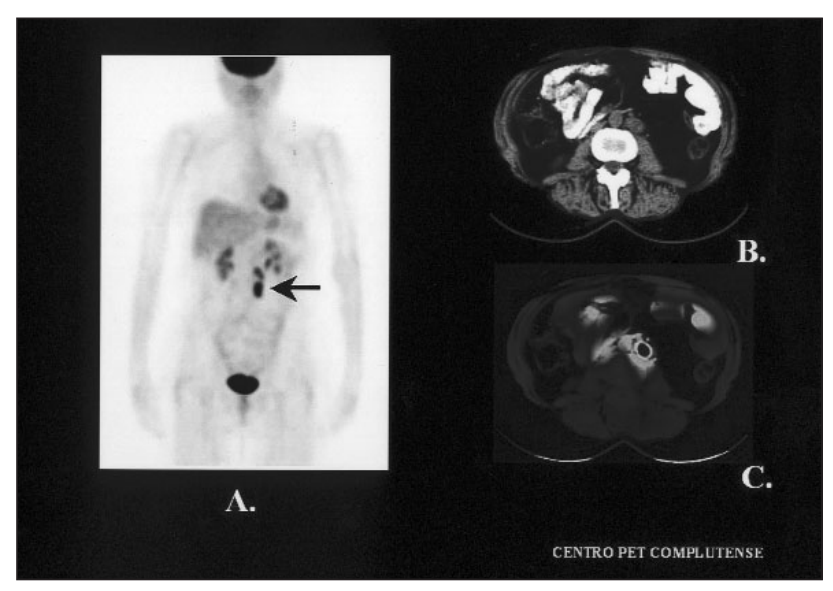

Figura 6. Paciente diagnosticada de adenocarcinoma endometrial tratado mediante cirugía y radioterapia. En TAC de control presenta adenopatía paraaórtica izquierda de dudoso significado. La PET muestra un elevado metabolismo de la misma, indicativo de metástasis ganglionar (A., flecha). Detalle del corte TAC transaxial a dicho nivel (B.) y de la fusión PET-TAC (C.).

trabajo de Umesaki y cols. ${ }^{76}$ demostró un $100 \%$ de sensibilidad en la detección de sarcomas uterinos en 5 pacientes, resultado superior a la RM $(80 \%)$ y a la ecografía (40\%). De manera general y al igual que ocurre con otras neoplasias pélvicas, la PET-FDG se ve limitada por la existencia de actividad fisiológica urinaria e intestinal de la zona. Además, debe tenerse en cuenta la posibilidad de que el útero presente una captación fisiológica más o menos acentuada en función del ciclo menstrual ${ }^{26}$. Más aún, los tumores benignos miometriales (leiomiomas) acumulan FDG con relativa frecuencia, en algunas ocasiones en grados similares a los tumores malignos. 
La utilidad de la PET en el diagnóstico de enfermedad residual o recurrente sólo ha sido evaluada en contados trabajos, aunque con resultados esperanzadores. Belhocine y cols. ${ }^{77}$ estudiaron 34 pacientes tratadas por carcinoma endometrial con sospecha de recidiva o como control post-terapia. Obtuvieron unas cifras de sensibilidad, especificidad, exactitud y valores predictivos positivo y negativo de $94 \%, 78 \%, 90 \%, 89 \%$ y $91 \%$, respectivamente, cambiando el manejo terapéutico en un tercio de los casos y descubriendo lesiones no sospechadas por otros métodos convencionales (Figs. 5 y 6). En otro estudio de Saga y cols. ${ }^{78}$ sobre 30 pacientes con sospecha de recurrencia de carcinoma endometrial, la PET mostró una sensibilidad, especifidad y exactitud de $100 \%$, $82 \%$ y $93 \%$, respectivamente, cifras superiores alas obtenidas por TAC y/o RM $(85 \%$, $86 \%$ y $85 \%$, respectivamente) y por los marcadores tumorales $(100 \%, 71 \%$ y $83 \%$, respectivamente). También se localizaron lesiones no sospechadas, con cambios en el manejo terapéutico en un tercio de las pacientes. Se necesitan más estudios que avalen los resultados preliminares obtenidos en estos trabajos.

\section{Conclusiones}

La PET con FDG tiene un valor limitado en el diagnóstico primario y estadificación del cáncer de ovario, aunque puede aportar información útil y completar los hallazgos de la radiología convencional. En pacientes sin evidencia de enfermedad tras el tratamiento primario, la PET no puede sustituir a la "second-look" dada su baja sensibilidad para detectar enfermedad micronodular. No obstante, en aquellos pacientes en los que se sospeche recurrencia por datos clínicos, de imagen o, sobre todo, por elevación de marcadores tumorales, la resolución de la PET es muy alta y debe considerarse en los casos de marcador CA-125 elevado y técnicas convencionales negativas. Los nuevos equipos híbridos PETTAC incluso mejoran el rendimiento diagnóstico y parece que esta técnica acabará imponiéndose a las cámaras PET dedicadas. El uso de la PET como instrumento para valorar la respuesta al tratamiento quimioterápico precisa de mayor estudio antes de su utilización de forma rutinaria.

En el cáncer de cérvix, la PET es una técnica útil en la estadificación pre-terapéutica de la enfermedad, ayudando una adecuada planificación tanto quirúrgica como radioterápica, con el impacto que ello conlleva en el manejo del paciente. El seguimiento con PET permite un diagnóstico precoz y exacto de la enfermedad recurrente, con resultados superiores a las técnicas convencionales. Aporta asimismo datos predictores en cuanto al pronóstico y a la respuesta a la terapia que pueden ser útiles para identificar aquellos pacientes que precisen de intervenciones más agresivas.

La aplicación de la PET en el cáncer de cuerpo uterino, aunque prometedora, necesita de mayor estudio para su validación.

\author{
Correspondencia: \\ Dr. J. P. Suárez Fernández \\ Centro PET Complutense \\ C/ Bartolomé Cossío, 10 \\ E-28040 Madrid \\ E-mail: pablosua@mixmail.com
}

\section{Bibliografía}

1. Whittemore AS, Harris R, Itnyre J. Characteristics relating to ovarian cancer risk: Collaborative analysis of 12 US case-control studies. Am J. Epidemiol 1992; 136: 1184-203.

2. Tornos C, Silva EG. Pathology of Epithelial Ovarian Cancer. Obst Gyn Clin N A 1994; 21: 63-78.

3. Rosenthal A, Jacobs I. Ovarian cancer screening. Semin Oncol 1998 Jun; 25(3): 315-25.

4. Disaia PJ, Creasman WT. Oncología ginecológica. Ed Mosby/Doyma: $4^{\mathrm{a}}$ ed, 1994:341-4.

5. Annual report on the results of treatment in gynecologic cancer (FIGO). Editado por F. Pettersson, Vol 21: Int J Gynecol and Obstet 1991; 36; suppl 1: 238-55.

6. International Union Against Cancer (UICC). TNM classification of malignant tumors, 5th edn. (1997). Wiley-Liss, New York, pp 87-90.

7. Pettersson F. Annual report on the results of treatment in gynecological cancer. Editorial Office Radiumhemmet. Stockholm, vol 22, 1994.

8. Walton LA, Yadusky A, Rubinstein L, Roth LM, Young MC. Stage II carcinoma of the ovary: An analysis of survival after comprehensive surgical staging adjuvant therapy. Gynecol Oncol 1992; 44: 55-60. 
9. Holschneider $\mathrm{CH}$, Berek JS. Ovarian cancer: epidemiology, biology, and pronostic factors. Semin Surg Oncol 2000; 19: 3-10.

10. Society of Gynecologic Oncologists: Clinical Practice Guidelines. Ovarian Cancer 1996; 1: 12-8.

11. Ozols RF and Vermorken JB. Chemotherapy of advanced ovarian cancer: Current status and future directions. Semin Oncol 1997; 1(Suppl 2): S2 1-9.

12. Kurokawa T, Yoshida Y, Kawahara K, et al. Expression of GLUT-1 glucose transfer, cellular proliferation activity and grade of tumor correlate with [F-18]-fluorodeoxyglucose uptake by positron emission tomography in epithelial tumors of the ovary. Int J Cancer 2004; 109: 926-32.

13. Bast RCJ, Xu FJ, Yu YH, Barnhill S, Zhang Z, Mills GB. CA 125: the past and the future. Int J Biol Markers 1998; 13: $179-87$

14. Sato S, Yokayama Y, Sakamoto T, Futagami M, Saito Y. Usefulness of mass screening for ovarian carcinoma using transvaginal ultrasonography. Cancer 2000; 89: 582-8.

15. Kawamoto S, Urban BA, Fishman EK. CT of epithelial ovarian tumors. Radiographics 1999; 19: S85-S102.

16. Rieber A, Nussle K, Stohr I, et al. Preoperative diagnosis of ovarian tumors with MR imaging: comparison with transvaginal sonography, positron emission tomography, and histologic findings. Am J Roentgenol 2001; 177: 1239.

17. Hubner KF, McDonald TW, Niethammerm JG, Smith GT, Gould HR, Buonocore E. Assessment of primary and metastasic ovarian cancer by positron emission tomography (PET) using 2-[18F]deoxyglucose (2-[18F]FDG). Gynecol Oncol 1993: 51: 197-204.

18. Römer W, Avril N, Dose J, et al. Metabolic characterization of ovarian tumors with positron-emission tomography and F-18 fluorodeoxyglucose. Rofo Fortschr Geb Rontgenstr Neuen Bildgeb Verfahr 1997; 166: 62-8.

19. Schröder W, Zimny M, Rudlowski C, Büll U, Rath W. The role of 18-F-fluoro-deoxyglucose positron emission tomography (18-F-FDG PET) in diagnosis of ovarian cancer. Int J Gynecol Cancer 1999; 9: 117-22.

20. Liebermann G, MacLean AB, Buscombe JR, et al. The clinical application of a dual head gamma camera with coincidence detection in 20 women with suspected ovarian cancer. BJOG 2001; 108: 1229-36.

21. Fenchel S, Grab D, Nuessle K, et al. Asymptomatic adnexal masses: correlation of FDG PET and histopathologic findings. Radiology 2002; 223: 780-8.

22. Zimny M. Ovarian cancer. En: Oehr P, Biersack HJ, Coleman RE, eds. PET and PET-CT in Oncology. Berlin Heidelberg: Springer-Verlag, Inc, 2004: 236.

23. Lapela ML, Leskinen-Kallio S, Varpula M, et al. Metabolic imaging of ovarian tumors with carbon-11-methionine: A PET study. J Nucl Med 1995; 36: 2196-200.

24. Zimny M, Schöder W, Wolters S, Cremerius U, Rath W, Büll U. 18F-fluorodeoxyglucose PET in ovarian carcinoma: methodology and preliminary results. Nuklearmedizin 1997; 36: 228-33.
25. Koyama K, Okamura T, Kawabe J, et al. Evaluation of 18F-FDG PET with bladder irrigation in patients with uterine and ovarian tumors. J Nucl Med 2003; 44: 353-8.

26. Lerman H, Metser U, Grisaru D, Fishman A, Lievshitz G, Even-Sapir E. Normal and abnormal 18F-FDG endometrial and ovarian uptake in pre and postmenopausal patients: assessment by PET/CT. J Nucl Med 2004; 45: 26671.

27. Torizuka T, Kanno T, Futatsubashi M, et al. Imaging of gynecologic tumors: comparison of (11)C-choline PET with (18)F-FDG PET. J Nucl Med 2003; 44: 1051-6.

28. Yoshida Y, Kurokawa T, Kawahara K, et al. Incremental benefits of FDG positron emission tomography over CT alone for the preoperative staging of ovarian cancer. AJR Am J Roentgenol 2004; 182: 227-33.

29. Drieskens O, Stroobants S, Gysen M, Vandenbosch G, Mortelmans L, Vergote I. Positron emission tomography with FDG in the detection of peritoneal and retroperitoneal metastases of ovarian cancer. Gynecol Obstet Invest 2003; 55: 130-4.

30. Kim EE, Lee MC, Inoue T, Wang WH. Gynecologic cancers. En: Clinical PET: Principles and applications. Kim SE, Chung JK, Kim EE., eds. New York: Springer-Verlag, Inc, 2004: 325-46.

31. Jimenez-Bonilla J, Maldonado A, Morales S, et al. Clinical impact of 18F-FDG-PET in the suspicion of recurrent ovarian carcinoma based on elevated tumor marker serum levels. Clin Positron Imaging 2000; 3: 231-6.

32. Rose PG, Faulhaber P, Miraldi F, Abdul-Karim FW. Positive emission tomography for evaluating a complete clinical response in patients with ovarian or peritoneal carcinoma: correlation with second-look laparotomy. Gynecol Oncol 2001; 82: 17-21.

33. Nakamoto Y, Saga T, Ishimori T, et al. Clinical value of positron emission tomography with FDG for recurrent ovarian cancer. AJR Am J Roentgenol 2001; 176: 1449-54.

34. Zimny M, Siggelkow W, Schroder W, et al. 2-[Fluorine18]-fluoro-2-deoxy-d-glucose positron emission tomography in the diagnosis of recurrent ovarian cancer. Gynecol Oncol 2001; 83: 310-5.

35. Torizuka T, Nobezawa S, Kanno T, et al. Ovarian cancer recurrence: role of whole-body positron emission tomography using 2-[fluorine-18]-fluoro-2-deoxy- D-glucose. Eur J Nucl Med Mol Imaging 2002; 29: 797-803.

36. Chang WC, Hung YC, Kao CH, Yen RF, Shen YY, Lin CC. Usefulness of whole body positron emission tomography (PET) with 18F-fluoro-2-deoxyglucose (FDG) to detect recurrent ovarian cancer based on asymptomatically elevated serum levels of tumor marker. Neoplasma 2002; 49: 329-33.

37. Cho SM, Ha HK, Byun JY, et al. Usefulness of FDG PET for assessment of early recurrent epithelial ovarian cancer. AJR Am J Roentgenol 2002; 179: 391-5.

38. Garcia MJ, Boan JF, Villar LM, Aramendia JM, Lopez G, Richter Echeverria JA. F-18-FDG positron emission tomography in the diagnosis of ovarian recurrence. Comparison 
with CT scan and CA 125. Rev Esp Med Nucl 2003; 22: 217-23.

39. Picchio M, Sironi S, Messa C, et al. Advanced ovarian carcinoma: usefulness of [(18)F]FDG-PET in combination with CT for lesion detection after primary treatment. Q J Nucl Med 2003; 47: 77-84.

40. Bristow RE, del Carmen MG, Pannu HK, et al. Clinically occult recurrent ovarian cancer: patient selection for secondary cytoreductive surgery using combined PET/CT. Gynecol Oncol 2003; 90: 519-28.

41. Sironi S, Messa C, Mangili G, et al. Integrated FDG PET/CT in patients with persistent ovarian cancer: correlation with histologic findings. Radiology 2004; 233: 433-40.

42. Smith GT, Hubner KF, McDonald T, et al. Cost analysis of FDG-PET for managing patients with ovarian cancer. Clin Positron Imaging 1999; 2: 63-70.

43. Scheidler J, Hricak H, Yu KK, Subak L, Segal MR. Radiological evaluation of lymph node metastases in patients with cervical cancer. A meta-analysis. JAMA 1997; 278: 1096-101.

44. Sugawara Y, Eisbruch A, Kosuda S, Recker BE, Kison PV, Wahl RL. Evaluation of FDG PET in patients with cervical cancer. J Nucl Med 1999; 40: 1125-31.

45. Rose PG, Adler LP, Rodriguez M, Faulhaber PF, AbdulKarim FW, Miraldi F. Positron emission tomography for evaluating para-aortic nodal metastasis in locally advanced cervical cancer before surgical staging: a surgicopathologic study. J Clin Oncol 1999; 17: 41-5.

46. Reinhardt MJ, Ehritt-Braun C, Vogelgesang D, et al. Metastatic lymph nodes in patients with cervical cancer: detection with MR imaging and FDG PET. Radiology 2001; 218: 776-82.

47. Narayan K, Hicks RJ, Jobling T, Bernshaw D, McKenzie AF.A comparison of MRI and PET scanning in surgically staged loco-regionally advanced cervical cancer: potential impact on treatment. Int J Gynecol Cancer 2001; 11: 263-71.

48. Yeh LS, Hung YC, Shen YY, Kao CH, Lin CC, Lee CC. Detecting para-aortic lymph nodal metastasis by positron emission tomography of $18 \mathrm{~F}$-fluorodeoxyglucose in advanced cervical cancer with negative magnetic resonance imaging findings. Oncol Rep 2002; 9: 1289-92.

49. Ma SY, See LC, Lai CH, et al. Delayed (18)F-FDG PET for detection of paraaortic lymph node metastases in cervical cancer patients. J Nucl Med 2003; 44: 1775-83.

50. Lin WC, Hung YC, Yeh LS, Kao CH, Yen RF, Shen YY. Usefulness of (18)F-fluorodeoxyglucose positron emission tomography to detect para-aortic lymph nodal metastasis in advanced cervical cancer with negative computed tomography findings. Gynecol Oncol 2003; 89: 73-6.

51. Reinhardt MJ. Cancer of the uterus. En: Oehr P, Biersack HJ, Coleman RE, eds. PET and PET-CT in Oncology. Berlin-Heidelberg: Springer-Verlag, Inc, 2004: 237-41.

52. Tran BN, Grigsby PW, Dehdashti F, Herzog TJ, Siegel BA. Occult supraclavicular lymph node metastasis identified by FDG-PET in patients with carcinoma of the uterine cervix. Gynecol Oncol 2003; 90: 572-6.
53. Kerr IG, Manji MF, Powe J, Bakheet S, Al Suhaibani, Subhi J. Positron emission tomography for the evaluation of metastases in patients with carcinoma of the cervix: a retrospective review. Gynecol Oncol 2001; 81: 477-80.

54. Malyapa RS, Mutic S, Low DA, et al. Physiologic FDGPET three-dimensional brachytherapy treatment planning for cervical cancer. Int J Radiat Oncol Biol Phys 2002; 54:1140-6

55. Mutic S, Malyapa RS, Grigsby PW, et al. PET-guided IMRT for cervical carcinoma with positive para-aortic lymph nodes-a dose-escalation treatment planning study. Int J Radiat Oncol Biol Phys 2003; 55: 28-35.

56. Esthappan J, Mutic S, Malyapa RS, et al. Treatment planning guidelines regarding the use of CT/PET-guided IMRT for cervical carcinoma with positive paraaortic lymph nodes. Int J Radiat Oncol Biol Phys 2004; 58: 1289-97.

57. Yen TC, Ng KK, Ma SY, et al. Value of dual-phase 2-fluoro-2-deoxy-d-glucose positron emission tomography in cervical cancer. J Clin Oncol 2003; 21: 3651-8.

58. Lai CH, Huang KG, See LC, et al. Restaging of recurrent cervical carcinoma with dual-phase [18F]fluoro-2-deoxyD-glucose positron emission tomography. Cancer 2004; 100: 544-52.

59. Park DH, Kim KH, Park SY, Lee BH, Choi CW, Chin SY. Diagnosis of recurrent uterine cervical cancer: computed tomography versus positron emission tomography. Korean J Radiol 2000; 1:51-5.

60. Sun SS, Chen TC, Yen RF, Shen YY, Changlai SP, Kao A. Value of whole body 18F-fluoro-2-deoxyglucose positron emission tomography in the evaluation of recurrent cervical cancer. Anticancer Res 2001; 21: 2957-61.

61. Ryu SY, Kim MH, Choi SC, Choi CW, Lee KH. Detection of early recurrence with $18 \mathrm{~F}$-FDG PET in patients with cervical cancer. J Nucl Med 2003; 44: 347-52.

62. Havrilesky LJ, Wong TZ, Secord AA, Berchuck A, ClarkePearson DL, Jones EL. The role of PET scanning in the detection of recurrent cervical cancer. Gynecol Oncol 2003; 90: $186-90$.

63. Lai CH, Huang KG, See LC, et al. Restaging of recurrent cervical carcinoma with dual-phase [18F]fluoro-2-deoxyD-glucose positron emission tomography. Cancer 2004; 100: 544-52.

64. Wong TZ, Jones EL, Coleman RE.Positron emission tomography with 2-deoxy-2-[(18)F]fluoro-D-glucose for evaluating local and distant disease in patients with cervical cancer. Mol Imaging Biol 2004; 6: 55-62.

65. Chang TC, Law KS, Hong JH, et al. Positron emission tomography for unexplained elevation of serum squamous cell carcinoma antigen levels during follow-up for patients with cervical malignancies: a phase II study. Cancer 2004; 101: 164-71.

66. Unger JB, Ivy JJ, Connor P, et al. Detection of recurrent cervical cancer by whole-body FDG PET scan in asymptomatic and symptomatic women. Gynecol Oncol 2004; 94: 212-6.

67. Hain S. PET imaging in testicular, ovarian and cervical 
cancer. En: Positron emission tomography: basic science and clinical practice. Valk PE, Bailey DL, Townsend DW, Maisey MN, eds. London: Springer-Verlag, Inc, 2003: 611-23.

68. Belhocine T, Thille A, Fridman V, et al. Contribution of whole-body 18FDG PET imaging in the management of cervical cancer. Gynecol Oncol 2002; 87: 90-7.

69. Yen TC, See LC, Chang TC, et al. Defining the priority of using 18F-FDG PET for recurrent cervical cancer. J Nucl Med. 2004; 45: 1632-9.

70. Giannopoulou C. The role of SPET and PET in monitoring tumor response to therapy. Eur J Nucl Med Mol Imaging 2003; 30: 1173-200.

71. Nakamoto Y, Eisbruch A, Achtyes ED, et al. Prognostic value of positron emission tomography using F-18-fluorodeoxyglucose in patients with cervical cancer undergoing radiotherapy. Gynecol Oncol 2002; 84: 289-95.

72. Grigsby PW, Siegel BA, Dehdashti F, Rader J, Zoberi I. Posttherapy $[18 \mathrm{~F}]$ fluorodeoxyglucose positron emission tomography in carcinoma of the cervix: response and outcome. J Clin Oncol 2004; 22: 2167-71.
73. Jang HJ, Lee KH, Kim YH, et al. The role of FDG-PET for predicting prognosis in squamous cell type uterine cervical carcinoma patients. J Nucl Med 2002; 43: 28.

74. Miller TR, Grigsby PW. Measurement of tumor volume by PET to evaluate prognosis in patients with advanced cervical cancer treated with radiation therapy. Int J Radiat Oncol Biol Phys 2002; 53: 353-9.

75. Grigsby PW, Siegel BA, Dehdashti F. Lymph node staging by positron emission tomography in patients with carcinoma of the cervix. J Clin Oncol 2001; 19: 3745-9.

76. Umesaki N, Tanada T, Miyama M, et al. Positron emission tomography $18 \mathrm{~F}$-fluorodeoxyglucose of uterine sarcoma: a comparison with magnetic resonance imaging and power doppler imaging. Gynecol Oncol 2001; 80: 372-7.

77. Belhocine T, De Barsy C, Hustinx R, Willems-Foidart J. Usefulness of (18)F-FDG PET in the post-therapy surveillance of endometrial carcinoma. Eur J Nucl Med Mol Imaging 2002; 29: 1132-9.

78. Saga $T$, Higashi $T$, Ishimori $T$, et al. Clinical value of FDG-PET in the follow up of post-operative patients with endometrial cancer. Ann Nucl Med 2003; 17: 197-203. 